OnLine Journal of Biological Sciences 8 (2): 44-48, 2008

ISSN 1608-4217

(C) 2008 Science Publications

\title{
Impairment of Retrograde Neuronal Transport in Cardiac Vagal Motoneurons in Streptozotocin-Induced Diabetic Rats: A Wheat Ger Agglutinin-Horseradish Peroxidase Neurohistochemical study
}

\author{
Odekunle A. and C.M. Phillips \\ Department of Preclinical Sciences, Faculty of Medical Sciences, Human Anatomy University, \\ The University of the West Indies, St. Augustine, Trinidad and Tobago, West Indies
}

\begin{abstract}
Central projections of vagal motoneurons to the heart were studied in diabetic rats using Wheat germ Agglutinin-Horseradish peroxidase (WGA-HRP). Experimental rats were rendered diabetic by intraperitoneal injection of streptozotocin in citrate buffer. The diabetic rats were maintained in a stable diabetic state by daily injection of insulin for 24 weeks. Age-matched control rats were injected intraperitoneally with citrate buffer not containing streptozotocin. Control rats were also kept alive for 24 weeks after citrate buffer injection. At the end of 24 weeks the two groups were prepared for injection with WGA-HRP. Following anesthesia with sodium pentobarbitone, thoracotomy was performed on the left aspect of the thorax to expose the heart. The atrial and ventricular walls were then injected with $5 \%$ WGA-HRP by multiple intramuscular penetrations. Experimental and control rats were sacrificed $72 \mathrm{~h}$ after tracer injection by transcardial perfusion first with normal saline followed by fixative and then buffered sucrose. Transverse serial frozen sections of the brainstem were then taken and processed for WGA-HRP neurohistochemistry and analyzed under light and dark-field microscopy. Analysis of the sections taken from diabetic rats revealed fewer WGA-HRP labeled neurons in the nucleus ambiguus (nA) than sections taken from control rats. Sporadic labeling of the dorsal motor nucleus of the vagus nerve was observed in control rat but not in the diabetic rats. It was concluded that the depletion of labeled neurons in the diabetic rats compared with the normoglycaemic rats is indicative of impairment of retrograde neuronal transport of WGAHRP in chronic diabetic state.
\end{abstract}

Key words: Diabetic rat, cardiac motoneurons, neuronal transport, horseradish peroxidase

\section{INTRODUCTION}

Diabetic-induced peripheral neuropathy is a significant complication resulting in increased patient morbidity and mortality. Several body systems are known to be implicated in this metabolic disorder in which cardiovascular complications are known to be the commonest causes of sudden death of patients. Visceral lesions associated with this disorder are often induced by disorders of the autonomic nervous system i.e., diabetic autonomic peripheral neuropathy which is commonly characterized by derangement in cardiovascular, gastrointestinal and respiratory systems $^{[1-4]}$.

Earlier studies have shown that vagal lesions in chronic STZ-induced diabetes were caused by hyperglycemia of diabetes ${ }^{[5,6]}$ and are associated with increased amount of metabolic markers which include glucose, fructose and sorbitol in the vagus nerve as well as abnormal plasma lipoprotein concentrations ${ }^{[7]}$. Cardiovascular dysfunctions associated with diabetes have been reported to include amongst others, microvascular lesions affecting the entire vasculature of the body, cardiac arrhythmias, impaired arterial baroreflex functions and orthostatic hypotension ${ }^{[1,8-10]}$.

Vagal lesions of chronic diabetes have also been shown to be accompanied by impairment in the neuronal transport capabilities of the vagus nerve $\mathrm{e}^{[5,11]}$. Whereas the impairment in the ability of the vagus nerve to transport target tissue-derived endogenous material such as NGF and NT-3 has been demonstrated in the diabetic rat, no change was recorded in the content of these materials in the vagally innervated organs studied ${ }^{[11]}$. Thus alteration in the concentration of tissue-derived materials cannot be held responsible for the observed impaired transportation in the nerve.

Corresponding Author: A. Odekunle, Department of Preclinical Sciences, Faculty of Medical Sciences, Human Anatomy University, The University of the West Indies, St. Augustine, Trinidad and Tobago, West Indies 
Since a healthy living neuron is actively involved in the transport of materials between the axonal terminals, dendrites and the cell body, it has been hypothesized that neuronal functional abnormalities such as observed in the diabetic state are accompanied by disruptions in the mechanism of neuronal transport in the affected vagus nerve. This hypothesis is supported by the demonstration of impairment of anterograde and retrograde transport of NGF, neurotrophins and the neuronal tracer, Fluorogold in the vagus nerve of STZ-induced chronic diabetic states ${ }^{[5,11]}$. Furthermore since investigations have shown that the reduction in the rate of neuronal transport of endogenous neurotrophins in the vagus nerve of streptozotocin-induced diabetic rats is not accompanied by reduction in the target tissue content of neurotrophins ${ }^{[11]}$, it is quite logical to infer that the impairment of neuronal transport is; (a) of neuronal origin and (b) impacted upon by the integrity of the cell bodies of origin of vagal nerve fibres.

While reduced neuronal transport in the diabetic rat could be due to impairment of transport mechanism, it could also emanate from loss of vagal neurons as a direct metabolic effect of hyperglycaemia of diabetes. The effects of diabetes on various organs innervated by the vagus nerve have been studied in various species $^{[4,6,11-15]}$.

To date there are very limited studies on the role of the vagus nerve in diabetic cardiopathy. The paucity of literature on this subject motivated the present study which was designed to determine the effect of STZinduced diabetes on the retrograde transport of a sensitive neuronal tracer, WGA-HRP by cardiac preganglionic parasympathetic vagal nerve fibres in the rat.

\section{MATERIALS AND METHODS}

Adult male and female Sprague-Dawley rats weight range between 200 and 300 gm were used for the study. These were divided into two groups (Experimental and Control). The experimental rats were injected intraperitoneally with STZ in a dosage of $60 \mathrm{mg} / \mathrm{kg}$ in 0.05 molar sodium citrate buffer at $\mathrm{pH} 4.5$ after light anaesthesia with ketamine/xylazine.

Twenty-four hours after STZ injection blood glucose levels were measured from tail-prick blood sample to confirm induction of hyperglycemia with blood glucose level of $>200 \mathrm{gm} \mathrm{dL}^{-1}$. The diabetic rats were subsequently treated with lent insulin at a daily dosage of 2-3IU/100G body weight to maintain a steady level of blood glucose between 200-300 gm dL $\mathrm{gm}^{-1}$ for a period of 24 weeks. Experimental rats' blood glucose level was monitored on a weekly basis throughout the duration of the experiment. Control rats were injected in the same manner as the experimental rat with the same volume $100 \mathrm{mM}$ of citrate phosphate buffer (not containing STZ), $\mathrm{pH}$ 4.5. Blood glucose level of control rats was also measured 24 hours after the injection to confirm a normoglyceamic level of $<200 \mathrm{mg} \mathrm{dL}^{-1}$. This was also measured weekly throughout the duration of the experiment to ascertain normoglycaemic status of control rats.

At the end of 24 weeks, six experimental and six control rats, which survived this phase of the experiment, were selected for treatment with WGAHRP. For surgical exposure of the heart, each rat was anaesthetized with an intraperitoneal injection of sodium pentobarbitone (Sagatal, May and Baker, Dabenham) in a dose of $60 \mathrm{mg} \mathrm{kg}^{-1}$ body weight. A left lateral thoracotomy along the third intercostal space was then performed to expose the sternocostal surface of the heart. With the aid of a 100 microlitre Hamilton syringe with a G31 gauge needle, $0.1 \mathrm{ml}$ of $5 \%$ WGAHRP in $0.5 \mathrm{M}$ sodium chloride was injected by multiple penetrations into the muscular coat of the atrial and ventricular walls of the heart care being taken to avoid injection of tracer into the cardiac cavities. Following each muscular injection, the injection site was pressed firmly with sterile gauze for $2 \mathrm{~min}$ to prevent tracer leakage into the pericardial sac.

After the injections the heart was wiped with sterile gauze and the thoracotomy incision closed in layers. The rats were then kept in a warm environment until recovery from anesthesia. Diabetic and control rats were anesthetized 48-72 $\mathrm{h}$ after tracer injection and subsequently perfused transcardially first with normal saline followed by a fixative containing $1 \%$ paraformaldehyde and $1.25 \%$ glutaraldehyde in $0.1 \mathrm{M}$ phosphate buffer, $\mathrm{pH} 7.4$ at room temperature and finally with $10 \%$ buffered sucrose at $4^{\circ} \mathrm{C}$.

Transverse serial frozen sections of the brainstem, $40 \mu$ in thickness were then taken and processed for WGA-HRP neurohistochemistry, using the modified Tetramethyl benzedene procedure recommended by Mesulam ${ }^{[16-17]}$. The sections were then analyzed under light and dark-field microscopy. Cell counting was done with the aid of a calibrated cell counting chamber. Only cells with distinct margin and in which the blueblack reaction products of TMB/WGA-HRP were seen were regarded labeled and counted.

\section{RESULTS}

Analysis of sections taken from the brainstem of diabetic and non-diabetic rats revealed the presence of 


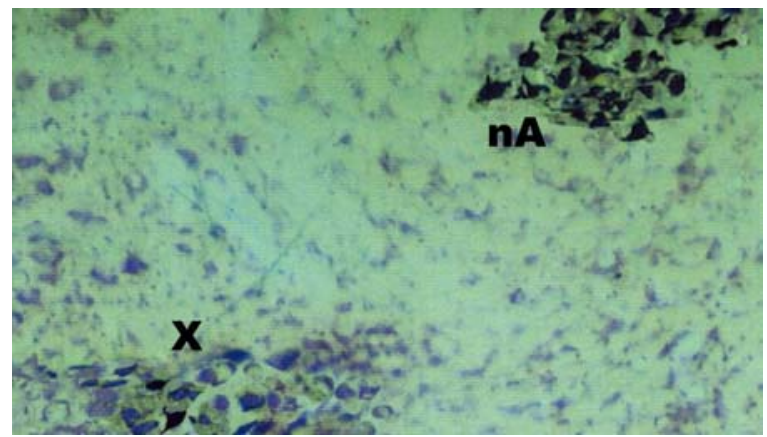

Fig. 1: Photomicrograph of a representative transverse section taken from the medulla oblongata of a non-diabetic (Control) rat, demonstrating WGAHRP labeled neurons (colored black) in the nucleus ambiguus following injections of WGAHRP into cardiac wall. (Observe the sparse labeling of the DMNV). nA. Nucleus ambiguous in the ventrolateral aspect of the medulla oblongata Dorsal motor nucleus of the vagus nerve $(\mathrm{DMNV})$. Magnification $=(\mathrm{X} 200)$.

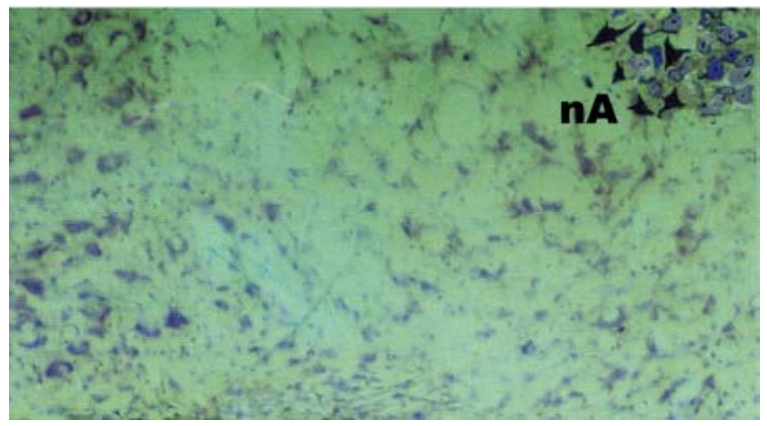

Fig. 2: Photomicrograph of a representative transverse section taken from the medulla oblongata of a STZ-induced diabetic rat demonstrating WGAHRP labeled neurons (colored black) in the nucleus ambiguus of the vagus nerve following injections of WGA-HRP into cardiac wall. Observe the reduction in the population of labeled neurons in this micrograph compared with Fig. 1. nA. Nucleus ambiguus in the ventrolateral aspect of the medulla oblongata. Magnification $=(\mathrm{X} 400)$.

Blue-black reaction products of WGA-HRP/TMB (Tetramethyl Benzedine) in neurons of the nucleus ambiguous (nA). Labeled neurons were bilaterally distributed along the entire rostrocaudal extent of the $\mathrm{nA}$ in both groups of rats (Fig. 1 and 2). The numbers of labeled neurons counted in the nA of the diabetic rats were also found to be significantly less than the number of labeled neurons counted in the non-diabetic rats. While the section containing the maximum number of labeled cells in the control rat had 50 labeled cells (left and right sides added) Fig. 1, comparable section in the diabetic rat had only 12 cells (Fig. 2). Sporadic labeling of neurons was also observed in the DMNV of controlled rats. While some sections had no labeled neuron at all, some had a maximum of two labeled neurons (Fig. 1). This nucleus was completely devoid of labeled neurons in the Diabetic rats.

\section{DISCUSSIONS}

The nucleus ambiguus in the medulla oblongata, one of the sources of vagal nerve fibres has been shown to project to the heart in several earlier studies in most animal species ${ }^{[18-30]}$. The robust labeling of this nucleus in the present study was therefore not surprising as it was anticipated and consistent with previous studies in healthy animal species. It is however very remarkable that fewer labeled neurons were observed in this nucleus in diabetic compared with non-diabetic rats. Since both groups of rats i.e., diabetic and non-diabetic were subjection to the same experimental procedures it appears logical to attribute the difference in the results of the present study to the diabetic state of the experimental rats. The result of the present study is also consistent with earlier reports in which depletion of labeled vagal neurons were observed following tracer injections of vagally innervated organs of diabetic rats $^{[11,31]}$. The result of the present study is also in agreement with that of Hellweg et al. and Lee et al. ${ }^{[5]}$.

Both groups of investigators had independently demonstrated impairment of neuronal transport of tissue-derived endogenous materials such as Nerve Growth Factor (NGF) and neurtrophin-3 (N-3) by the vagus nerve in diabetic rats. Both groups of authors had also implicated impairment of neuronal transport in the pathogenesis of diabetic neuropathy.

Several neurotrophic mechanisms and axonal transport systems of the neuron are implicated in the transport of neuronal tracer by neurons. Derangement of these systems by the metabolic disorder associated with chronic diabetes could be responsible for the depletion of labeled vagal neurons observed in the diabetic rats in the present study.

The depleted labeling observed in this and previous studies could also have arisen from the reduction in the availability of the injected tracer to the nerve terminal either as a result of degeneration of vagal efferent terminals or localized cardiac lesions associated with diabetes $^{[1,8,9]}$. On the other hand, diabetic-induced impairment of uptake of the tracer through the 
processes of endocytosis and internalization could also have been responsible for the impairment of retrograde transport of tracer observed in the present study. Furthermore, alterations in sorbitol-polyol pathway and/or oxidative stress which have been demonstrated to be responsible for impairment of neuronal transport in other nerves ${ }^{[11,32-35]}$ may also account for the observed impairment of neuronal transport in the vagus nerve in the present report.

Neurofilaments/tubulin components of the neuron which are critical to axonal transport could also be directly affected by the hyperglycaemia associated with diabetes or indirectly by the impairment of access to neurotrophins in the diabetic state ${ }^{[1,36-37]}$. An earlier electron microscopic study by Tay and Wong has also demonstrated degenerative changes in the DMNV in diabetic rats ${ }^{[6]}$. Thus direct neuronal loss in the nucleus ambiguus could not be ruled out as the likely cause of the depletion in the number of labeled neurons in the present study.

The weak labeling of the DMNV in the control rat and the total absence of labeled neurons in the diabetic in the study are quite consisted with the information obtained from earlier studies of healthy experimental animal species which had all indicated that the $\mathrm{nA}$ is the principal source of cardiac vagal fibres ${ }^{[23,26]}$.

\section{CONCLUSION}

In conclusion, the present study has provided evidence of impaired neuronal transport in cardiac vagal nerve in diabetic rat and suggested underlying mechanisms of the impaired neuronal function. Further investigations at the peripheral and central ends of the vagal system are required to determine the precise mechanisms of the defective neuronal functions.

\section{ACKNOWLEDGEMENT}

We are deeply grateful to The Campus Research and Publication Fund Committee, University of the West Indies, St. Augustine Campus and The Caribbean Health Research Council for the grants made available to us for this study. We also sincerely appreciate the technical assistance offered by Ms N. Homer and Ms C. Lord in the Anatomy Research Laboratory.

\section{REFERENCES}

1. Bernarde, L., 2000. Clinical evaluation of arterial baroreflex activity in diabetes. Diabetes Nutr. Metab., 13: 331-340.
2. Kautzner, J. and A.J. Camm, 1997. Clinical relevance of heart rate variabilitry. Clin. Cardiol., 20: $162-168$.

3. Pieron, M., A.J. Scheen, J.L. Corhey, M.F. Radermecker and P.J. Lefebvre, 1997. Bronchial reactivity in diabetic patients. Rev. Mal. Respir., 14: 379-385.

4. Koch, K.T., 1999. Diabetic gastropathy: Gastric neuromuscular dysfunction in diabetes mellitus: A review of symptoms, pathophysiology and treatment. Dig. Dis. Sci., 44: 1061-1075.

5. Lee, P.G., C.H. Thomas, F. Cai, J. Regalia and C.J. Helke, 2001. Streptozotocin-induced diabetes causes metabolic changes and altrations in neurotrophin contents and retrograde transport in the cervical vagus nerve. Exp. Neurol., 170: 149-161.

6. Tay, S.S. and W.C. Wong, 1994. Short- and longterm effects of STZ-induced diabetes on the dorsal motor nucleus of the vagus nerve in the rat. Acta Anat., 150: 274-281.

7. Zimmermann, J., L. Schramm, E. Mulzer, E. Heidbreder, H.A. Henrich and C. Wanner, 1997. Cardiovascular risk factors in in diabetic nephropathy. Med. Klin. (Munich) 92: 74-78.

8. Britland, S.T., R.J. Young, A.K. Sharma, D. Lee, A.K. AH-See and B.F. Clarke, 1990. Vagus nerve morphology in diabetic gastropathy. Diabet. Med., 7: 780-787.

9. Burger, A.J. and D. Aronson, 2001. Effect of diabetes mellitus on heart rate variability in patients with congestive heart failure. Pacing Clin. Electrophysiol., 24: 53-59.

10. Valensi, P. J.P. Huard, C. Giroux and J.R. Attali, 1997. Factors involved in cardiac autonomic neuropathy in diabetic patients. J. Diabet. Complicat., 11: 180-187.

11. Lee, P.G., F. Cai and C.J. Helke, 2002. Streptozotocin-induced diabetes reduces retrograde axonal transport in the afferent and efferent vagus nerve. Brain Res., 941: 127-136.

12. Hellweg, R. and H.D. Hartung, 1990. Edogenous levels of Nerve Growth Factor (NGF) are altered in experimental diabetes mellitus: A possible role for NGF in the pathogenesis of diabetic neuropathy. J. Neurosci. Res., 26: 258-267.

13. Kristensson, K., C. Nordborg, Y. Olsson and P. Sourander, 1971. Changes in the vagus nerve in diabetes mellitus. Acta. Pathol. Microbiol. Scand., 79: 684-685.

14. Guo, Y.P., J.G. McLeod and J. Baverstock, 1987. Pathological changes in the vagus nerve in diabetes and chronic alcoholism. J. Neurol. Neurosurg. Psychiatry, 50: 1449-1453. 
15. Lee, P., H. Zhue and C.J. Helke, 2001. Axotomy alters neurotrophin and neurotrophin receptors mRNAs in the vagus nerve and nodose ganglion of the rat. Brain Res. Mol. Brain Res., 87: 31-41.

16. Mesulam, M.M., 1978. TMB for HRP neurohistochemistry: A non-carcinogenic blue reaction-product with superior sensitivity for visualizing neural afferents and efferents. J. Histochem. Cytochem., 26: 106-117.

17. Mesulam, M.M., 1982. Tracing Neural Connections with HRP. A Wiley Interscience Publication, New York, pp: 1-152.

18. Getz, B. and T. Sirnes, 1949. Localization within the dorsal motor vagal nucleus: An experimental investigation. J. Comp. Neurol., 90: 95-110.

19. Mitchell, G.A.C. and R. Warwick, 1955. The dorsal vagal nucleus. Acta Anatomical, 25: 371-395.

20. Miller, F.R. and J.J. Bowmn, 1961. The cardioiniiibitory centre. Am. J. Physiol., 39: 149-153.

21. Calaresu, F.R. and M.K. Cottle, 1965. Origin of cardiomotor fibres in the dorsal motor nucleus in the cat: A histological study. J. Physiol., 176: 252-260.

22. Calaresu, F.R. and J.W. Pearce, 1965. Effect on heart rate of electrical stimulation of medullary vagal structures in the cat. J. Physiol., 176: 241-25 1.

23. Todo, K., T. Yamamoto, H. Satomi, H. Ise, H. Takatama and K. Takahashi, 1977. Origin of vagal preganglionic fibres to the sino atrial and atrioventricular node regions in the cat heart as studied by HRP method. Brain Res., 130: 545-550.

24. Schwaber, J.S. and D.H. Cohen, 1978. Field potential and single unit analyses of avian dorsal motor nucleus of the vagus and the criteria for identifying vagal cardiac cells of origin. Brain Res., 147: 79-90.

25. Nosaka, S., T. Yamamoto and K. Yasumaga, 1979. Localization of vagal cardioinhibitory preganglionic neurons within the rat brain. $J$. Comp. Neurol., 186: 79-92.

26. Geis, G.C. and R.D. Wurster, 1980. Horseradish peroxidase localization of cardiac vagal preganglionic somata. Brain Res., 182: 19-30.

27. Ciriello, J. and F.R. Calaresu, 1982. Medullary origin of vagal preganglionic axons to the heart of the cat. J Auton. Nerv. Syst. 5: 9-22.
28. Panneton, W.M., P.F. McCulloch, Y. Tan and P. Yavari, 1996. Brainstem origin of preganglionic cardiac motoneurons in the muskrat. Brain Res., 738: 342-346.

29. Hsieh, J.H., R.F. Chen, J.J. Wu, C.T. Yen and C.Y. Chai, 1998. Vagal innervation of the gastrointestinal tract arises from dorsal motor nucleus while that of the heart largely from the nucleus ambiguus in the cat. J. Auton. Nerv. Syst., 70: 38-50.

30. Odekunle, A. and T.I. Chinnah, 2002. The localization of cardiac vagal motoneurons in the medulla of the Ferret (Mustela Putorius Furo). J Caribb. Vet. Med. Assoc., 2: 12-17.

31. Odekunle, A., 2006. Impairment of transneuronal traffic in streptozotocin-induced diabetes, a WGAHRP neurohistochemical study in the rat. J. Biomed. Sci., 13: 675-680.

32. Mayer, J.H. and D.R. Tomlinson, 1983. Axonal transport of cholinergic transmitter enzymes in vagus and sciatic nerves of rats with acute experimental diabetes mellitus; correlation with motor nerve conduction velocity and effects of insulin. Neuroscience, 9: 951-957.

33. Dahlin, L.B., D.R. Archer and W.G. Mclean, 1989. Treatment with an aldose reductase inhibitor can reduce the susceptibility of fast axonal transport following nerve compression in the streptozotocindiabetic rat. Diabetologia, 30: 414-418.

34. Germani, E., E. Lesma, A.M. Di Giulio and A. Gorio, 1999. Progressive and selective changes in neurotrophin factor expression and substance $p$ axonal transport induced by perinatal diabetes: protective action of antioxidant treatment. J. Neurosci. Res., 57: 521-528.

35. Ino, U.M., L. Zhang, H. Naka, H. Kuriyama and M. Yamamoto, 2000. Polyol metabolism of retrograde axonal transport in diabetic rat large optic nerve fibre. Invest. Ophthalmol. Vis. Sci., 41: 4055-4058.

36. Yagihashi, S., M. Kamijo and K. Watanabe, 1990. Reduced myelinated fiber size correlates with loss of axonal neurofilaments in peripheral nerve of chronically streptozotocin diabetic rats. Am. J. Pathol., 136: 1365-1373.

37. Tomlinson, D.R., P. Fernyhough, L.T. Diemel and K. Maeda, 1996. Deficient neurotrophic support in the aetiology of diabetic neuropathy. Diabet. Med., 13: 679-681. 